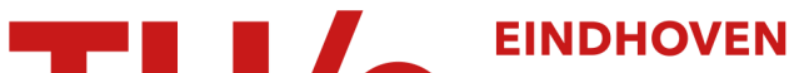 \\ UNIVERSITY OF \\ TECHNOLOGY
}

\section{Dielectric structures with bound modes for microcavity lasers}

\author{
Citation for published version (APA): \\ Visser, P. M., Allaart, K., \& Lenstra, D. (2002). Dielectric structures with bound modes for microcavity lasers. \\ Physical Review E - Statistical, Nonlinear, and Soft Matter Physics, 65(5), 056604-1/11. [056604]. \\ https://doi.org/10.1103/PhysRevE.65.056604
}

DOI:

10.1103/PhysRevE.65.056604

Document status and date:

Published: 01/01/2002

\section{Document Version:}

Publisher's PDF, also known as Version of Record (includes final page, issue and volume numbers)

\section{Please check the document version of this publication:}

- A submitted manuscript is the version of the article upon submission and before peer-review. There can be important differences between the submitted version and the official published version of record. People interested in the research are advised to contact the author for the final version of the publication, or visit the $\mathrm{DOI}$ to the publisher's website.

- The final author version and the galley proof are versions of the publication after peer review.

- The final published version features the final layout of the paper including the volume, issue and page numbers.

Link to publication

\section{General rights}

Copyright and moral rights for the publications made accessible in the public portal are retained by the authors and/or other copyright owners and it is a condition of accessing publications that users recognise and abide by the legal requirements associated with these rights.

- Users may download and print one copy of any publication from the public portal for the purpose of private study or research.

- You may not further distribute the material or use it for any profit-making activity or commercial gain

- You may freely distribute the URL identifying the publication in the public portal.

If the publication is distributed under the terms of Article 25fa of the Dutch Copyright Act, indicated by the "Taverne" license above, please follow below link for the End User Agreement:

www.tue.nl/taverne

Take down policy

If you believe that this document breaches copyright please contact us at:

openaccess@tue.nl

providing details and we will investigate your claim. 


\title{
Dielectric structures with bound modes for microcavity lasers
}

\author{
P. M. Visser, ${ }^{*}$ K. Allaart, ${ }^{\dagger}$ and D. Lenstra ${ }^{\ddagger}$ \\ Vrije Universiteit Amsterdam, De Boelelaan 1081, 1081HV Amsterdam, The Netherlands
}

(Received 21 November 2001; published 29 April 2002)

\begin{abstract}
Cavity modes of dielectric microspheres and vertical cavity surface emitting lasers, in spite of their high $Q$, are never exactly bound, but have a finite width due to leakage at the borders. We propose types of microstructures that sustain three-dimensionally bound modes of the radiation field when dissipation is neglected. Unlike photonic crystals, the photonic systems that we consider here rely on periodicity in only one or two dimensions. In particular, we discuss a cavity composed of two crossed vertical layers combined with a periodic structure of horizontal layers. The layers have an anisotropic dielectric tensor, which could be obtained by making air holes in the vertical and horizontal directions within isotropic material. We calculate cavity resonance frequencies and spontaneous emission rates. The simplicity of this laser geometry allows an analytical study of light propagation and amplification in three dimensions.
\end{abstract}

DOI: 10.1103/PhysRevE.65.056604

PACS number(s): 42.60.-v, 32.80.-t, 78.67.-n

\section{INTRODUCTION}

Vertical cavity surface emitting lasers (VCSELs) are examples of very small lasers $[1,2]$. These microstructures can have narrow cavity resonances as a result of the localizing effect of the cylindrical waveguide in combination with the Bragg reflection in the stacked disks. The long lifetime of photons created from the recombination of electron-hole pairs in the central layer makes the stimulated emission efficient. At present, the efficiency of VCSELs is mainly limited by leakage at the borders. There is loss of radiation through spontaneous emission which exits from the side. Also, the light in the cavity mode decays because evanescent waves are not reflected in the vertical direction. The reflection conditions of the interior guided wave and the outer evanescent wave do not match, which gives rise to losses at the boundary and determines the width of the cavity resonances. Exact bound modes do not occur in VCSELs. When the coupling to the lasing mode is enhanced by making the system (and thereby the mode volume) smaller, the evanescent fields become more important and the lifetime of the mode decreases. This kind of incompatibility between small mode volume and high finesse occurs in whispering gallery modes of microspheres [3-6] too, also because of losses at the boundary.

Exact bound states for the radiative field can occur in spatially infinite dielectric structures, for example in photonic crystals. When such a crystal has a point defect, it is possible to create a bound state at a frequency inside a threedimensional photonic band gap $[7,8]$. In the absence of dissipation, the state has an infinite lifetime while propagating solutions do not exist at the frequency of the bound mode. Anderson localization in a disordered structure provides another means to create bound states of light [9-11]. A twolevel atom coupled to a localized field mode would make a perfect realization of the Jaynes-Cummings model [12].

In this paper we consider a class of systems, other than

\footnotetext{
*Electronic address: PMV@nat.VU.nl

†Electronic address: Allaart@nat.vu.nl

‡Electronic address: Lenstra@nat.VU.nl
}

photonic crystals and disordered structures, where localized modes occur. These systems are periodic in one or two directions only. There is no three-dimensional band gap and spontaneous emission is possible at the same frequencies as the bound states. Because the modes are small and lossless, systems with such a design may prove useful in future semiconductor microlasers with strong coupling and low threshold. The geometry of the structures is simple, but the layers must satisfy specific requirements. In Sec. II we specify the class of systems, composed of anisotropic dielectric material, where bound states occur. The layers must have a principal axis of lower dielectric constant in the vertical direction for the vertical layers, and in the horizontal layers for the horizontal layers. This can be achieved by drilling air holes in isotropic material of high dielectric constant. An even more favorable situation would occur when the air holes would be filled with material with a negative dynamical dielectric constant. Sections III and IV provide two examples of a waveguide and a cavity which, in a thin layer approximation, are analytically solvable. We then show how the bound solutions arise in the cavity. The partial spontaneous emission rates in the different types of radiative and guided modes are calculated in Sec. V.

\section{STRUCTURES THAT SUPPORT BOUND STATES}

\section{A. Required form of the dielectric tensor}

The bound states arise in structures with specific polarization properties. The dielectric tensor of the class of these solvable configurations is given by

$$
\boldsymbol{\varepsilon}(\vec{r})=[1+\hat{z} \hat{z} U(x)+\hat{z} \hat{z} V(y)+(1-\hat{z} \hat{z}) W(z)] \varepsilon_{1} .
$$

Here the functions $U(x), V(y)$, and $W(z)$ describe structures of layers normal to the $x, y$, and $z$ directions. The background medium, in which the functions $U, V$, and $W$ are zero, is isotropic, given by the dielectric constant $\varepsilon_{1}$. For air $\varepsilon_{1} \approx 1$, but we have in mind a background made of dielectric material and $\varepsilon_{1}>1$. In this paper, we consider real $\varepsilon_{1}$ and real functions $U, V$, and $W$, so that the entire system is lossless. The case with lossy layers and an active gain medium is 
studied elsewhere [13]. The structures shown in Figs. 1 and 2 are examples that will be discussed in Secs. III and IV.

The optical properties of dielectric structures, like spontaneous emission, amplification, and loss, can be calculated from a complete set of modes of the electromagnetic field. These field modes are the orthogonal solutions of Maxwell's equation for a stationary electric field of frequency $\omega$ in a medium with relative dielectric function $\boldsymbol{\varepsilon}(\vec{r})$ :

$$
\vec{\nabla} \times \vec{\nabla} \times \vec{E}=(\omega / c)^{2} \boldsymbol{\varepsilon}(\vec{r}) \vec{E} .
$$

When the dielectric tensor is of the form given in Eq. (1), two types of solution can be discerned: those with the $\vec{H}$ field in the $x y$ plane, hereafter called the $s$-type modes, and those with the $\vec{E}$ field in the $x y$ plane, hereafter called the $p$ type. This nomenclature is adopted in view of the stack of parallel layers in Fig. 2. The $p$-type solutions are insensitive to the functions $U$ and $V$ in Eq. (1); they are therefore not localized in the $x$ or $y$ direction. The $s$-type modes can be expressed as

$$
\begin{gathered}
\vec{E}(\vec{r})=\frac{1}{\sqrt{\mathcal{R}}}\left(\frac{k_{z}^{2}}{k^{2}} \vec{\nabla}-\hat{z} \frac{d}{d z}\right) f(x) g(y) h(z), \\
\mathcal{R}=\left|k^{2}-k_{z}^{2}\right|\left|k_{z}^{2}\right| \varepsilon_{1} / k^{2},
\end{gathered}
$$

in terms of the scalar functions $f(x), g(y)$, and $h(z)$. Here $\mathcal{R}$ is a normalization constant and $k_{z}, k$ are eigenvalues in differential equations for the functions $f, g$, and $h$. This can be proven by direct substitution in Maxwell's equation. As only the second term of Eq. (3) survives, when $\vec{\nabla} \times \vec{\nabla} \times$ is applied, one finds immediately

$$
\vec{\nabla} \times \vec{\nabla} \times \vec{E}=\frac{1}{\sqrt{\mathcal{R}}}\left(\vec{\nabla}^{2} \hat{z}-\vec{\nabla} \frac{d}{d z}\right) \frac{d}{d z} f(x) g(y) h(z) .
$$

The substitution of Eqs. (1) and (4) in Eq. (2) gives separate scalar equations for the functions $f(x), g(y)$, and $h(z)$ :

$$
\begin{gathered}
-\frac{d^{2}}{d x^{2}} f(x)=k_{x}^{2} f(x)+\left(k^{2}-k_{z}^{2}\right) U(x) f(x), \\
-\frac{d^{2}}{d y^{2}} g(y)=k_{y}^{2} g(y)+\left(k^{2}-k_{z}^{2}\right) V(y) g(y), \\
-\frac{d^{2}}{d z^{2}} h(z)=k_{z}^{2} h(z)+k_{z}^{2} W(z) h(x) .
\end{gathered}
$$

The three eigenvalues are related by

$$
k_{x}^{2}+k_{y}^{2}+k_{z}^{2}=k^{2}=(\omega / c)^{2} \varepsilon_{1} .
$$

In the regions where $U, V$, and $W$ are zero, the functions $f, g$, and $h$ are superpositions of plane waves and the total field is a superposition of eight plane waves with the wave vectors $\vec{k}= \pm \hat{x} k_{x} \pm \hat{y} k_{y} \pm \hat{z} k_{z}$. The polarization vector of each plane wave is proportional to $\vec{k} \times \vec{k} \times \hat{z}$. In the case of a lossless medium, considered here, the components $k_{x}, k_{y}, k_{z}$ must be real or purely imaginary. It follows from Eq. (8) that at least one of these wave vector components must be real.

Bound states in structures described with a dielectric tensor of the form of Eq. (1) are found when Eqs. (5)-(7) allow simultaneously localized solutions for $f, g$, and $h$. This will be the case for specific choices of the functions $U, V$, and $W$. It follows from standard wave mechanics that a localized solution in a potential of finite extent is found if the potential is attractive and allows a negative eigenvalue $k_{x}^{2}, k_{y}^{2}$, or $k_{z}^{2}$ above the potential minimum. For an extended structure, localized solutions occur when the potential is periodic in two half spaces. In that case, the corresponding eigenvalue $k_{x}^{2}$, $k_{y}^{2}$, or $k_{z}^{2}$ is positive and one needs a discrete solution inside a band gap. Structures with bound states can be designed by combining these localizing effects. Because at least one of the eigenvalues $k_{x}^{2}, k_{y}^{2}$, or $k_{z}^{2}$ must be positive, periodicity in at least one dimension is needed.

The effective potential $W(z)$ in Eq. (7) is multiplied by the eigenvalue $k_{z}^{2}$, so that a localized solution for $h(z)$ is found if $W(z)<-1$ in some finite region. This implies that the structure described by $W$ must have a negative dielectric constant for the relevant frequency domain. The effective potentials in Eqs. (5) and (6) have a prefactor $k^{2}-k_{z}^{2}=k_{x}^{2}$ $+k_{y}^{2}$. A localized solution for $f$ (or $g$ ) can be found in this case, when this prefactor and the potential are both positive or both negative. For the case $k_{x}^{2}+k_{y}^{2}>0$ the solution can only be localized in either the $x$ or the $y$ direction, not in both. In case $k_{x}^{2}+k_{y}^{2}<0$, solutions can in principle be localized in one or two directions with a finite structure.

From these considerations we conclude that the following structures will sustain bound states: (1) Periodic vertical structures in one direction, so that $U$ or $V$ is periodic in two half spaces, combined with a horizontal structure with negative dielectric constant, so that $W(z)<-1$ in a finite region. (2) Periodic horizontal structures combined with low index vertical structures, so that $U(x)<-1 / 2$ and $V(y)<-1 / 2$. (This is the type of structure that we consider in the following sections.) (3) A two-dimensional (2D) periodic structure with periodicity in one of the two vertical directions and also in the horizontal direction. (4) All three functions $U, V$, and $W$ periodic. Then one obtains a solvable model for a $3 \mathrm{D}$ photonic crystal.

\section{B. Normalization of the modes}

The full vector solutions given in Eq. (3) in terms of the scalar wave functions $f, g$, and $h$ must be normalized with the standard normalization condition

$$
\int d^{3} \vec{r} \vec{E}_{\vec{k}^{\prime}}^{*}(\vec{r}) \boldsymbol{\varepsilon}(\vec{r}) \vec{E}_{\vec{k}}(\vec{r})=\delta^{3}\left(\vec{k}^{\prime}-\vec{k}\right) .
$$

The dielectric tensor $\varepsilon$ appears in this expression, because it is the vector field $\sqrt{\varepsilon} \vec{E}$ that is an eigensolution of a Hermitian operator $[14,15]$. This expression is strictly speaking only valid when all three components of the wave vector belong to continuous parts of the eigenvalue spectra. When discrete solutions occur, we will replace the individual components of the 3D $\delta$ function with a Kronecker $\delta$. The required normal- 
ization of Eq. (9) can be obtained with the following choice of normalization for the scalar wave functions $f, g$, and $h$ in Eqs. (5)-(7):

$$
\begin{aligned}
\int_{-\infty}^{\infty} d x \int_{-\infty}^{\infty} d y f_{k_{x}^{\prime}}^{*}(x) f_{k_{x}}(x) g_{k_{y}^{\prime}}^{*}(y) g_{k_{y}}(y) \\
\times[1+U(x)+V(y)] \\
=\delta\left(k_{x}^{\prime}-k_{x}\right) \delta\left(k_{y}^{\prime}-k_{y}\right) \\
\int_{-\infty}^{\infty} d z h_{k_{z}^{\prime}}^{*}(z) h_{k_{z}}(z)[1+W(z)]=\delta\left(k_{z}^{\prime}-k_{z}\right) .
\end{aligned}
$$

If we assume that $U, V$, and $W$ are nonzero in a finite region only, the density of states is independent of the components $k_{x}, k_{y}, k_{z}$ of the wave vector of an incident plane wave. In that case, the effect of the potentials on the normalization of extended wave functions is negligible and the normalizations can be written as

$$
\begin{aligned}
& \int_{-\infty}^{\infty} d x f_{k_{x}^{\prime}}^{*}(x) f_{k_{x}}(x)=\delta\left(k_{x}^{\prime}-k_{x}\right), \\
& \int_{-\infty}^{\infty} d y g_{k_{y}^{\prime}}^{*}(y) g_{k_{y}}(y)=\delta\left(k_{y}^{\prime}-k_{y}\right) .
\end{aligned}
$$

The respective normalizations for localized wave functions are

$$
\begin{gathered}
\int_{-\infty}^{\infty} d x\left|f_{k_{x}}(x)\right|^{2}[1+U(x)]=1, \\
\int_{-\infty}^{\infty} d y\left|g_{k_{y}}(y)\right|^{2}[1+V(y)]=1, \\
\int_{-\infty}^{\infty} d x \int_{-\infty}^{\infty} d y\left|f_{k_{x}}(x) g_{k_{y}}(y)\right|^{2}[1+U(x)+V(y)]=1,
\end{gathered}
$$

in case either $k_{x}$ or $k_{y}$ or both are discrete eigenvalues. We will also consider systems that are periodic everywhere or periodic in a half space for the $z$ coordinate, so that $W(z)$ extends over an infinite region. The density of states is constant in the quasimomentum $p$ for an incoming Bloch state. In this case the wave functions $h(p, z)$ in one energy band must be normalized according to

$$
\int_{-\infty}^{\infty} d z h^{*}\left(p^{\prime}, z\right) h(p, z)[1+W(z)]=\delta\left(p^{\prime}-p\right)
$$

The normalization of Bloch states is explained in more detail in Appendix A. Properties of the $p$-type modes that are needed for the calculation of spontaneous emission rates in Section V are discussed in Appendix B.

\section{WAVEGUIDE OF CROSSED LAYERS}

One example of interest is the system of two crossed vertical planes depicted in Fig. 1. This configuration is described with Eq. (1) and the choice

$$
U(x)=\theta(d-2|x|) \chi / d,
$$

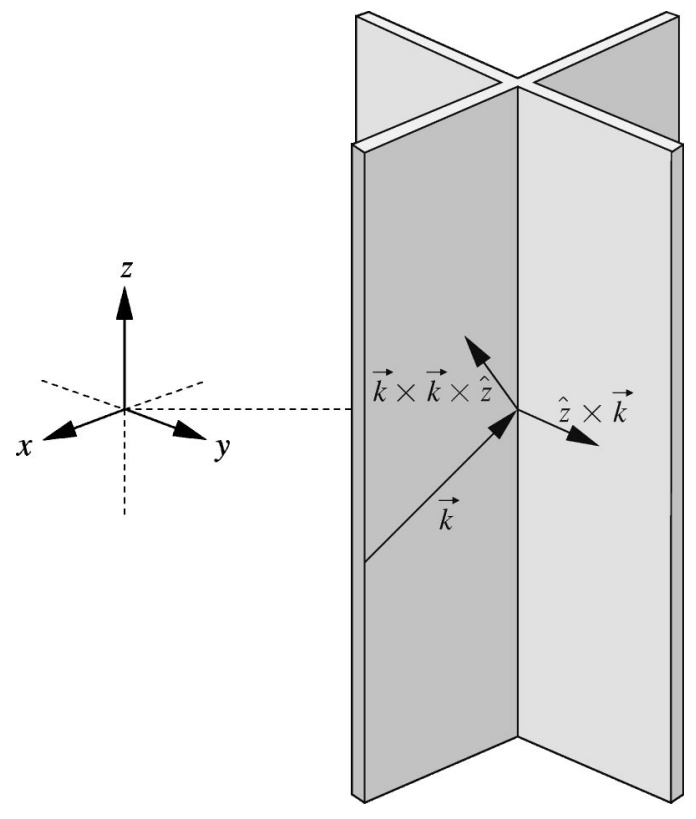

FIG. 1. A waveguide of crossed planes of anisotropic material with a principal axis in the $z$ direction. For any incident plane wave with wave vector $\vec{k}$ the vectors $\hat{z} \times \vec{k}$ and $\vec{k} \times \vec{k} \times \hat{z}$ are eigenpolarizations of reflection and transmission. This is illustrated here for the case that $\vec{k}$ lies in the $x z$ plane.

$$
V(y)=\theta(d-2|y|) \chi / d .
$$

Here $\theta(x)$ is the step function. The effective susceptibility $\chi$ is given by $\chi=\left(\varepsilon_{2}-\varepsilon_{1}\right) d / \varepsilon_{1}$. The functions $U$ and $V$ describe dielectric layers with thickness $d$ along the vertical $y z$ and $x z$ planes and $W(z)=0$. Inside these layers the dielectric tensor is $\varepsilon=(1-\hat{z} \hat{z}) \varepsilon_{1}+\hat{z} \hat{z} \varepsilon_{2}$. If the layers are characterized by a dielectric constant higher than the background, one has $\varepsilon_{2}>\varepsilon_{1}$ and then $\chi$ is positive; if the structures have a lower dielectric constant, then $\varepsilon_{2}<\varepsilon_{1}$ and $\chi$ is negative.

The propagating and guided waves in this structure follow from Eqs. (5) - (7). It is clear that Eq. (7) for the behavior in the $z$ direction allows a simple plane wave solution $h(z)$ $=\exp \left(i k_{z} z\right)$. Because the effective potentials $U(x)$ and $V(y)$ in this equation are even functions, one can consider solutions that are either even or odd in $x$ and $y$. If $k_{x}$ is real, the even and odd solutions, in the thin layer approximation $[16,17]$, are

$$
\begin{gathered}
f^{+}(x)=\sqrt{1 / \pi} \cos \left(k_{x}|x|+\phi_{x}\right), \\
f^{-}(x)=\sqrt{1 / \pi} \sin k_{x} x .
\end{gathered}
$$

The even solutions are characterized by a phase shift $\phi_{x}$. The value follows from the condition that $f(x)$ is continuous and differentiable at the borders of the layers. In the limit of small layer thickness, the phase shift calculated from Eq. (5) is given by

$$
\tan \phi_{x}=\kappa / k_{x}, \quad \kappa=k_{x}^{2} d / 2+\left(k^{2}-k_{z}^{2}\right) \chi / 2 .
$$

Localized solutions follow from the requirement that $k_{x}$ is imaginary. For a thin layer, one localized solution with $k_{x}$ 
$=i \kappa$ exists, when $\kappa$ is positive and given by Eq. (16). The corresponding normalized wave function is

$$
\begin{gathered}
f(x)=\sqrt{\kappa /|1+\chi \kappa|} \exp (-\kappa|x|), \\
\kappa=\left[\sqrt{1+\left(k^{2}-k_{z}^{2}\right) \chi d}-1\right] / d .
\end{gathered}
$$

The even, the odd, and the bound solutions form a complete set for the eigenvalue equation (5). The normalization of the continuum solutions is given by Eq. (10).

Combinations of the localized and propagating solutions for $f(x)$ and $g(y)$ give field modes that are propagating in three dimensions, in two dimensions, or in one dimension. In the following we will call these different types 3D, 2D, and 1D modes. For each of these types, the range of values of $k_{z}$ for a fixed wave number $k$ will generally be different. The allowed value for $k_{z}$ for the $3 \mathrm{D}$ modes is

$$
k_{z}^{2}=k^{2}-k_{x}^{2}-k_{y}^{2} \leqslant k^{2} .
$$

When $\chi>0$, only one of the two functions $f(x)$ or $g(y)$ can be localized, because $k^{2}-k_{z}^{2}=k_{x}^{2}+k_{y}^{2}$ must be positive. The region of allowed values for $k_{z}$ of the 2D modes is therefore also given by Eq. (19).

When $\chi<0$, the functions $U$ and $V$ in Eqs. (5) and (6) are negative. Only when $\chi<-d / 2$ can this result in localized solutions. Then both $f(x)$ and $g(y)$ need to be localized at the same time. This demonstrates that the crossed planes can act as a waveguide for some $s$-type modes. In the thin layer limit, the 1D modes are determined by the wave functions $g(y)=f(y)$ and Eq. (17) and the wave vector components are

$$
\begin{gathered}
k_{x}=k_{y}=i \kappa=2 i /(2|\chi|-d), \\
k_{z}^{2}=k^{2}+2 \kappa^{2}=k^{2}+8 /(2|\chi|-d)^{2} .
\end{gathered}
$$

The behavior in the overlap regions (where both $|x|<d / 2$ and $|y|<d / 2$ ) can be neglected only for thin layers: $d \ll|\chi|$. For the existence of 1D modes, this implies that $\chi$ and the dielectric constant $\varepsilon_{2}$ must be negative. On the other hand, if $\varepsilon_{2}$ is not negative, it is important that the structure in the overlap region must also be described with the form (1), which is $\varepsilon=(1-\hat{z} \hat{z}) \varepsilon_{1}+\hat{z} \hat{z}\left(2 \varepsilon_{2}-\varepsilon_{1}\right)$. One finds that the $z$ component of this tensor $2 \varepsilon_{2}-\varepsilon_{1}=(2 \chi / d+1) \varepsilon_{1}$ must be negative. Strictly speaking, the waveguide is perfect only when material with a negative dielectric constant is used.

For $\chi$ in the interval between $-d / 2$ and 0 , localized solutions for $f(x)$ or $g(y)$ are not possible. When $\chi$ lies in the interval between $-d$ and $-d / 2$ there exist 1D modes but no 2D modes. When $\chi<-d$ there exist both 1D and 2D modes. The spectral region for the $2 \mathrm{D}$ modes is given by

$$
k_{z}^{2}=k^{2}-k_{x}^{2}-k_{y}^{2} \geqslant k^{2}+4 /(|\chi|-d)^{2} .
$$

\section{A CAVITY AND ITS BOUND STATES}

We will now consider the system depicted in Fig. 2. This system basically consists of the waveguide of Fig. 1 discussed in Sec. III, supplemented with a structure of horizon- tal layers. Bragg reflection in these horizontal layers can suppress the propagation in the $z$ direction. By leaving out the central layer, the periodicity is interrupted at $z=0$, which results in wave functions that are localized in the region around the defect. The fully bound states will arise as combinations of the localized solutions for $f(x), g(y)$, and $h(z)$. For these modes, both $k_{x}$ and $k_{y}$ are imaginary and $k_{z}$ is a discrete but real solution inside a band gap.

The horizontal layers in the structure of Fig. 2 have width $b$ and their spacing, center to center, is given by the parameter $a$. The functions $U, V$, and $W$ in Eq. (1) that describe the system are given by Eqs. (13) and (14), and

$$
\begin{aligned}
W(z)= & \sum_{l=-\infty}^{\infty} \theta(b-2|z-l a|) \xi / b \\
& +\theta(b-2|z|)(\alpha-\xi) / b .
\end{aligned}
$$

This function is plotted in Fig. 3. With the effective susceptibility $\xi=\left(\varepsilon_{2}-\varepsilon_{1}\right) b / \varepsilon_{1}$, the dielectric tensor inside the horizontal layers is $\varepsilon=\hat{z} \hat{z} \varepsilon_{1}+(1-\hat{z} \hat{z}) \varepsilon_{2}$. For the cavity with bound states the central layer is absent and $\alpha=0$. We will also discuss the periodic case, in the presence of a central layer when $\alpha=\xi$. In this paper we consider layers that are infinite in extension and number. The bound states decay exponentially in all spatial directions, and are therefore good approximations to a physical realization of finite size. The circular shape of the horizontal planes in Fig. 2 is therefore also not essential. The entire structure, with the required anisotropy, can be created when the vertical layers are formed by vertical air holes (of subwavelength diameter), and the horizontal layers are formed by horizontal air holes in the $x$ and $y$ directions.

\section{A. Band structure for the propagating modes}

Before we discuss the case $\alpha=0$ in Eq. (23), we first study Eq. (7) for $h(z)$ with a fully periodic potential $W(z)$.

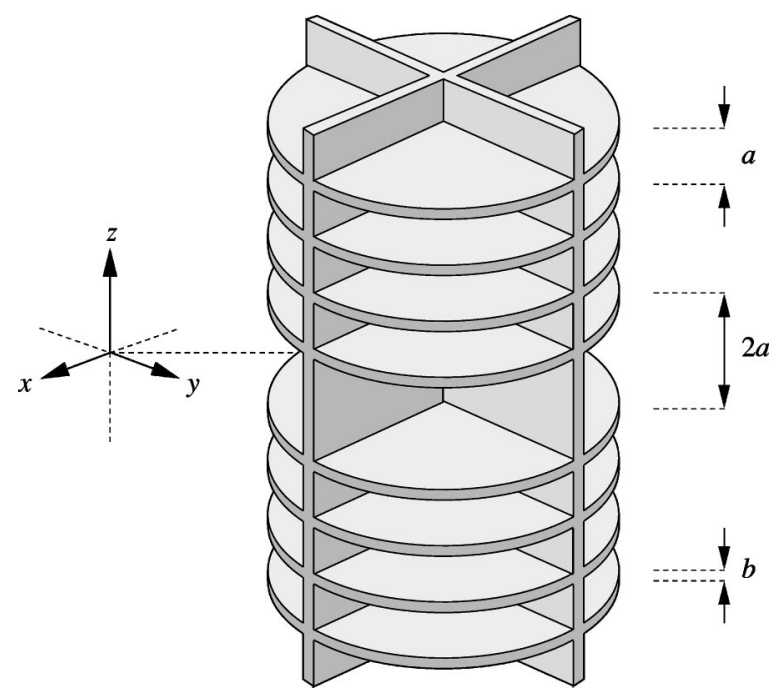

FIG. 2. Proposed structure for a cavity with exact bound modes. The dielectric tensor that characterizes the layers has a specific anisotropy given by Eq. (1). The corresponding dielectric constant must be lower than that of the background. 


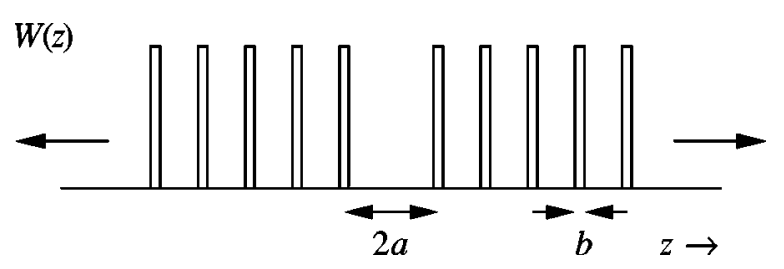

FIG. 3. The function $W(z)$, plotted here for $\alpha=0$, acts as an effective potential for the wave function $h(z)$. It is periodic in the half spaces left and right from $z=0$ with period $a$. The absence of a layer at $z=0$ creates localized waves.

This situation, realized when $\alpha=\xi$, is interesting because it describes the behavior of electromagnetic fields away from the central region. The Bloch solutions of the periodic onedimensional system, which we shall denote by $h_{n}(p, z)$, are defined by the requirement that the states obtain a phase factor $e^{i p a}$ upon translation over one period $a$. We have for integer $l$,

$$
h_{n}(p, z+l a)=e^{i l p a} h_{n}(p, z)
$$

Here the discrete index $n=1,2, \ldots$ is the band number and $p$ is the quasimomentum. The quasimomentum $p$ is a periodic variable modulo $q=2 \pi / a$ and one may choose $p$ to be in the first Brillouin zone $[-q / 2, q / 2]$. A few relevant properties for the normalization of Bloch waves are discussed in Appendix A. In the region $b / 2 \leqslant z \leqslant a-b / 2$ between two layers, the Bloch waves can be written as a sum of two plane waves: $h_{n}(p, z)=c_{1} e^{i k_{z} z}+c_{2} e^{-i k_{z} z}$, with coefficients $c_{1}$ and $c_{2}$. For thin layers, the requirement that the wave function is continuous at $z=0$ and $z=a$, in combination with the Bloch condition (24), leads to the form

$$
\begin{aligned}
h_{n}(p, z)= & \frac{1}{\sqrt{2 \pi R_{n}(p)} \sin k_{z} a} \\
& \times\left[e^{i p a} \sin k_{z} z+\sin k_{z}(a-z)\right],
\end{aligned}
$$

with normalization constant $R_{n}(p)$. The behavior of $h_{n}(p, z)$ for the other values of $z$ is determined with Eq. (24). The relationship between the quasimomentum $p$ and the wave vector $k_{z}$ now derives from the behavior at the layers. At the position of the layers the derivative of $h_{n}(p, z)$ makes a step of $-\xi k_{z}^{2} h_{n}(p, 0)$. This gives the well known relation [18]

$$
\cos p a=\cos \left(k_{z} a+\phi_{z}\right) / \cos \phi_{z} .
$$

Here the phase $\phi_{z}$ is the phase shift of a single layer, defined by

$$
\tan \phi_{z}=\xi k_{z} / 2, \quad-\pi / 2<\phi_{z}<\pi / 2 .
$$

The normalization of $h_{n}(p, z)$ is determined by Eq. (12). With Eqs. (26) and (27), the normalization constant in Eq. (25) is found to be

$$
R_{n}(p)=1+\left(\frac{1}{\tan k_{z} a}+\frac{1}{k_{z} a}\right) \tan \phi_{z} .
$$

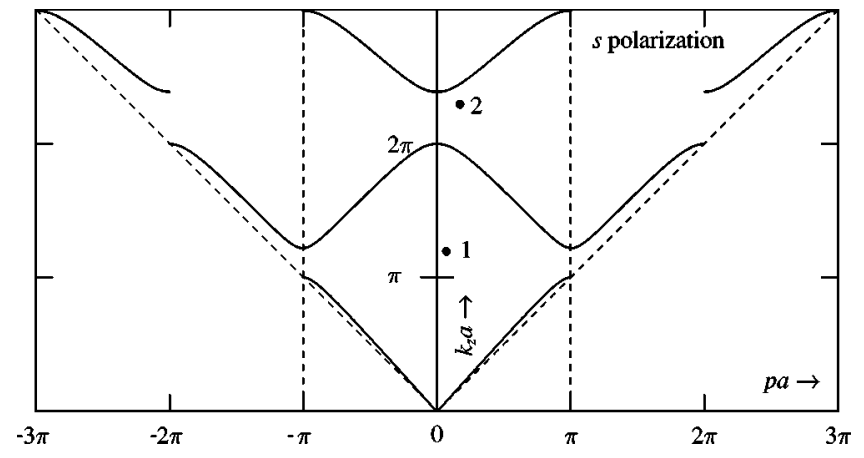

FIG. 4. Band structure of the $s$-type modes. The eigenvalue $k_{z}$ is plotted as a function of the quasimomentum $p$ for the first three Brillouin zones, as given by Eq. (29) for $\xi=-3 a / 16$. Discrete solutions inside the band gaps are represented as the points $\left(\lambda_{n}, k_{z n}\right)$, of which the first two are indicated. Here $\lambda_{n}=\operatorname{Im} p_{n}$.

From Eq. (26), one can obtain the standard form of a dispersion relation with the wave vector $k_{z}$, expressed as a function of the band index $n$ and the Bloch momentum $p$. This gives

$$
k_{z n}(p) a=2 \pi \operatorname{int}(n / 2)-(-)^{n} \arccos \left(\cos \phi_{z} \cos p a\right)-\phi_{z} .
$$

The resulting band structure is plotted in Fig. 4. For odd $n$ the minimum is found for $p=0$, and the maximum for $p$ $=q / 2$; for even $n$ the minimum is at $p=q / 2$ and the maximum is at $p=0$. It follows that the energy bands for $k_{z}$ are the intervals

$$
\begin{gathered}
(n-1) \pi \leqslant k_{z} a \leqslant n \pi-2 \phi_{z} \text { if } \xi>0, \\
(n-1) \pi+2\left|\phi_{z}\right| \leqslant k_{z} a \leqslant n \pi \text { if } \xi<0 .
\end{gathered}
$$

Hence the width of the band gaps is $2\left|\phi_{z}\right| / a$. The expressions (29) and (30) are not explicit, however, because the phase $\phi_{z}$ depends on $k_{z}$ through Eq. (27).

\section{B. The discrete cavity modes}

When $W(z)$ is given by Eq. (23) with $\alpha=0$, the periodicity is interrupted at $z=0$. We shall now describe how to obtain the propagating and localized solutions for $\alpha=0$. When $\chi<-d / 2$, the localized solution for $f(x)$ and $g(y)$ can be combined with a localized solution of $h(z)$ in Eq. (3) to give a fully bound state.

Although $W(z)$ is not periodic for $\alpha \neq \xi$, the stationary states can be expressed in the Bloch solutions of the case $\alpha=\xi$. In fact, a solution with an eigenvalue $k_{z}$ in the energy band can always be written as a superposition $h_{n}^{+}(p, z)$ $=c_{1} h_{n}(p, z)+c_{2} h_{n}(-p, z)$ of the two Bloch waves with positive and negative quasimomentum in the region $z \geqslant b / 2$. This obviously can also be done for the region $z \leqslant-b / 2$, but with different coefficients $c_{1}$ and $c_{2}$. Because at $z=0$ there is no layer, the values of the coefficients are determined by the condition that the solution is continuous and differentiable at $z=0$. The band structure of the periodic lattice thus remains intact. Because the potential $W(z)$ is even, one can consider modes that are even and odd functions of $z$. The even modes 
are fixed by the condition $(d / d z) h_{n}^{+}(p, 0)=0$, which gives $c_{1}=c_{2}^{*}=e^{-i \phi} / \sqrt{2}$ with

$$
\tan \phi=\tan \phi_{z} \sin k_{z} a / \sin p a .
$$

The odd modes are fixed by the condition $h_{n}^{-}(p, 0)=0$, which gives $c_{1}=-c_{2}=1 / \sqrt{2}$. The even and odd propagating solutions can be seen as a superposition of two scattering solutions by the lattice defect at $z=0$ in a further periodic system that have incoming Bloch waves from the left and from the right side of $z=0$. The mode density therefore is the same as the mode density for the Bloch states of the periodic case. The solutions $h_{n}^{+}(p, z)$ and $h_{n}^{-}(p, z)$ are also normalized with Eq. (12).

Bound modes occur for values of $k_{z}$ inside the band gaps. These modes are characterized by an imaginary quasimomentum of the form $p_{n}=i \lambda_{n}+n \pi / a$. Here $n=1,2,3, \ldots$ is the number that counts the energy band gaps. The fact that the quasimomentum is complex implies that Eq. (24) becomes

$$
h_{n}(z+a)=(-)^{n} e^{-\lambda_{n} a} h_{n}(z)
$$

for $z \geqslant b / 2$. Hence, the bound modes are localized in the $z$ direction near the lattice defect at $z=0$ and decay exponentially with decay constant $\lambda_{n}$. For thin layers, the presence or absence of the central layer has no effect on solutions that are odd functions of $z$. Therefore, only localized solutions that are even functions in $z$ occur. The two boundary conditions are obtained by substitution of $p=i \lambda+n \pi / a$ in the eigenvalue equation (26) and taking $(d h / d z)(0)=0$ for the solution given by Eq. (25). This gives the following two equations for $p$ and $k_{z}$ :

$$
\begin{aligned}
(-)^{n} e^{-\lambda a} & =\cos k_{z} a \\
& =(-)^{n} \cosh \lambda a-\tan \phi_{z} \sin k_{z} a .
\end{aligned}
$$

By elimination of $\lambda$, the relation between $k_{z}$ and $k$ for the discrete solutions inside the $n$th band gap is found to be

$$
\tan \left(n \pi-k_{z} a\right)=2 \tan \phi_{z} .
$$

When Eqs. (24) and (31) are substituted in Eq. (25), the corresponding localized wave function becomes

$$
h_{n}(z)=\frac{1}{\sqrt{R_{n}}}\left(\cos k_{z n} a\right)^{l(z)} \cos k_{z n}[|z|-l(z) a] .
$$

Here $l(z)=\operatorname{int}(|z| / a)$ is the number of layers between position $z$ and 0 , and $k_{z n}$ is the $n$th solution of Eq. (32). The wave function decays exponentially in both the positive and negative $z$ directions. The normalization constant in Eq. (33) is given by

$$
R_{n}=\left(\frac{1}{\sin ^{2} k_{z n} a}-\frac{1}{k_{z n} a \tan k_{z n} a}\right) a .
$$

To second order in $\xi$, Eqs. (27) and (32) give $\lambda_{n}$ $=(n \pi \xi)^{2} / 2 a^{3}, k_{z n}=n \pi /(a+\xi)$. For positive (negative) $\xi$, the top (bottom) of the energy bands lies at $k_{z}=n \pi(a$ $-\xi) / a^{2}$. The propagation vector $k_{z n}$ of a localized mode thus

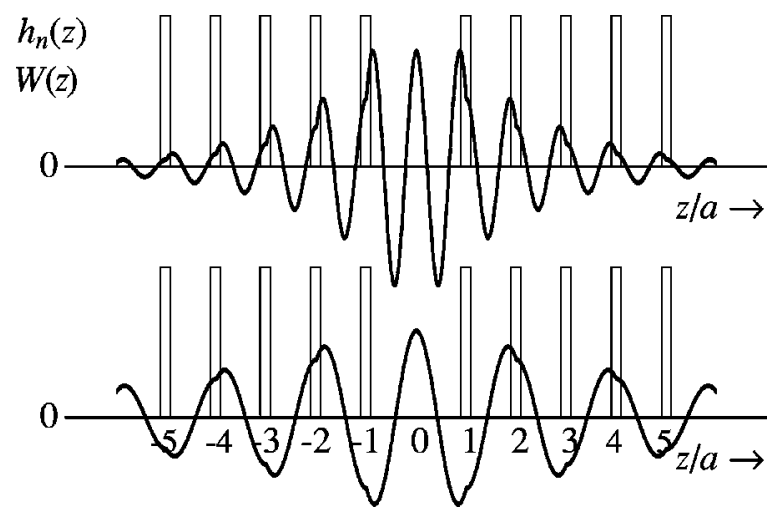

FIG. 5. Spatial wave functions $h_{n}(z)$ of the bound states along the $z$ direction, as given by Eq. (33). From bottom to top: the first two states $n=1,2$, for $\xi=-3 a / 16$. Kinks occur at the positions of the planes, and not at $z=0$.

lies only slightly above (below) the energy bands. The discrete eigenvalues $\lambda_{n}$ and the corresponding values $k_{z n}$ are indicated by the dots in Fig. 4. A few localized wave functions are plotted in Fig. 5.

The fully bound states are obtained from the localized solutions for $f(x), g(y)=f(y)$, and $h_{n}(z)$ as given by Eqs. (17) and (33), with the wave vector components $k_{x}, k_{y}, k_{z n}$ given by Eq. (20) and a discrete solution of Eq. (32). Substitution of these functions in the general vector solution given by Eq. (3) gives the explicit expression

$$
\begin{aligned}
\vec{E}_{n}(\vec{r})= & \frac{\kappa}{\sqrt{|1+2 \chi \kappa| R_{n}}} e^{-\kappa|x|-\kappa|y|}\left(\cos k_{z} a\right)^{l(z)} \\
& \times\left[\left(\frac{\hat{x} x}{|x|}+\frac{\hat{y} y}{|y|}\right) \cos \left[k_{z}|z|-l(z) a\right]\right. \\
& \left.+\frac{2 \kappa \hat{z} z}{k_{z}|z|} \sin \left[k_{z}|z|-l(z) a\right]\right]
\end{aligned}
$$

The intensity at the cavity center is $\vec{E}_{n}^{2}(\overrightarrow{0})=8 /\left(4 \chi^{2}\right.$ $\left.-d^{2}\right) R_{n}$. For the moderate numerical values $\chi=-3 d / 4$, $\xi$ $=-3 a / 16, d=a$, an effective mode volume of approximately 9.7 cubic optical wavelengths is found. The cavity must have at least 10 layers to sustain a bound state of this size.

The frequency of a bound state $\omega=c k \varepsilon_{1}^{-1 / 2}$ is obtained from Eq. (21) with a solution $k_{z n}$ of Eq. (32). Usually, one wants to fix the physical dimensions $a, b, d$ of the microcavity, so that the lowest order bound state $(n=1)$ is resonant with a given frequency $\omega$. These cavity resonances might be difficult to excite, because they are extremely narrow. It follows from Eqs. (21) and (32) that the optimal choice of the lattice spacing $a$ for $n=1$ is

$$
a=\frac{\pi-\arctan \left[\xi \sqrt{k^{2}+2 \kappa^{2}}\right]}{\sqrt{k^{2}+2 \kappa^{2}}},
$$




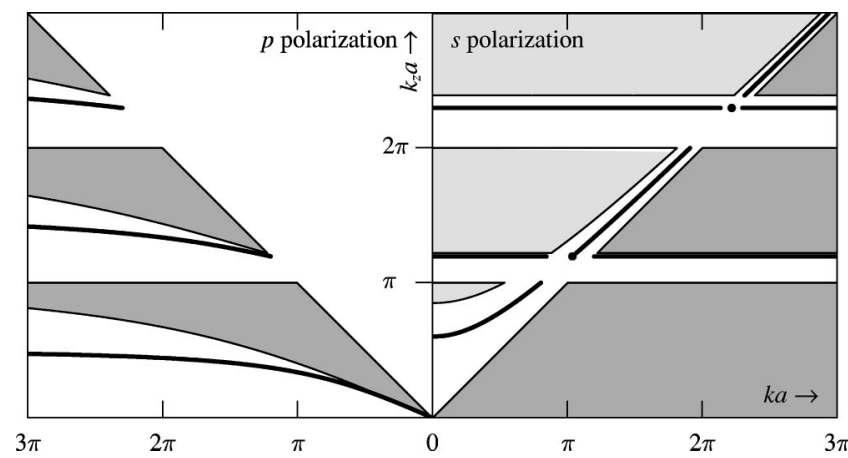

FIG. 6. Mode structure for $\chi=-3 a / 4, \xi=-3 a / 16$. The eigenvalue $k_{z}$ is plotted as a function of the wave number $k$. On the left side, solutions of $p$-type polarization; on the right side, the $s$ type. Dark and light shaded regions are 3D modes and 2D modes, respectively. Horizontal and curved lines are 2D modes localized in the $z$ direction only, and 1D modes localized in two of the three directions, respectively. Isolated points are bound states. The light shaded regions are absent when $\chi>-d$. Lines and points above $k_{z}=k$ are absent when $\chi>-d / 2$.

with $k=\varepsilon_{1}^{1 / 2} \omega / c$. If $|\xi|$ is small, then $a=\pi / k$ so that a full wavelength fits between the middle two layers. If $|\xi|$ is big, the layer to layer distance approaches $a=\pi / 2 k_{z}$.

The eigenvalue spectrum is plotted in Fig. 6 . The effect of localization in the $z$ direction is seen as lines inside the band gaps for $k_{z}$. In this figure we adopted $\chi=-3 a / 4, \xi=$ $-3 a / 16$, which could correspond to the situation of $\varepsilon_{1}=4$, $\varepsilon_{2}=1$ for cavity sizes $d=a=4 b$. The bound states are clearly shown as isolated points. The different mode types and their degree of localization are tabulated in Fig. 8 in Appendix B.

\section{SPONTANEOUS EMISSION}

The rich variety of the field modes in the cavity discussed in the previous section will show up in the angular dependence of spontaneous emission from an emitter placed inside the structure. For instance, the fraction of light emitted along the $z$ axis is predominantly given by emission in the $s$ mode that is localized in the $x$ and $y$ directions. In this section we calculate the partial spontaneous emission rates for a dipole placed at the origin of the cavity, into the different types of radiation modes (listed in Fig. 8 in Appendix B). This provides information about the spatial directions of the emitted radiation below the lasing threshold. The total rate of spontaneous emission [19-22] is the sum of these contributions and differs from the free-space emission rate.

Consider emission into field modes propagating in three dimensions. If the dipole moment is $\vec{\mu}=\mu \hat{\mu}$, oriented in direction $\hat{\mu}$, the emission rate according to Fermi's golden rule is given by

$$
\begin{aligned}
\frac{\Gamma_{x y z}}{\Gamma_{\mathrm{bg}}}= & \frac{3 \pi^{2} \varepsilon_{1}}{k^{2}} \sum \int_{0}^{\infty} d k_{x} \int_{0}^{\infty} d k_{y} \\
& \times \int_{0}^{\infty} d p \delta(k-|\vec{k}|)\left|\hat{\mu} \cdot \vec{E}_{\vec{k}}(\overrightarrow{0})\right|^{2} .
\end{aligned}
$$

We denote with $\Gamma_{\mathrm{bg}}=12 k^{3} \mu^{2} \varepsilon_{1}^{5 / 2} / \hbar\left(2 \varepsilon_{1}+1\right)^{2}$ the spontaneous emission rate in the background medium in Gaussian units [14]. The summation is over the polarization types $s, p$ and the eight possibilities of parity (even, odd in $x, y, z$ ), for which the indices are suppressed. When the emission into a localized mode is calculated, for instance one that is bound in the $x$ direction, the integral over $k_{x}$ is replaced by the discrete sum and the modes with imaginary $k_{x}=i \kappa$ are substituted. We now calculate the partial emission rates for a dipole located at the origin, with dipole moments in the $z$ and in the $x$ directions. From these, the emission rate of a dipole with arbitrary orientation can be determined.

The required electric field amplitudes at the cavity center are calculated in Appendix C. We assume here that $\chi$ lies in the interval between $-d$ and $-d / 2$, which is physically the most interesting case. Then there are three-dimensional radiative modes (labeled $x y z$, as listed in Fig. 8), modes localized in the vertical direction $(x y)$, modes guided by the two vertical planes $(z)$, and bound states (0). For an atom with a transition dipole moment oriented in the vertical direction $\hat{\mu}=\hat{z}$, the partial emission rates are

$$
\begin{gathered}
\frac{\Gamma_{x y z}}{\Gamma_{\mathrm{bg}}}=\frac{3}{2} \int_{0}^{k} d k_{z} \frac{k^{2}-k_{z}^{2}}{k^{3}} \frac{\left(\sqrt{k^{2}+\kappa^{2}-k_{z}^{2}}-\kappa\right)^{2}}{k^{2}+2 \kappa^{2}-k_{z}^{2}} \sigma, \\
\Gamma_{x y}=0, \\
\frac{\Gamma_{z}}{\Gamma_{\mathrm{bg}}}=\frac{24 \pi \kappa^{2}}{\left(4 \chi^{2}-d^{2}\right) k^{3} k_{1}} \sigma\left(k_{1}\right), \quad \Gamma_{0}=0 .
\end{gathered}
$$

The partial emission rates for an atom with dipole moment in the direction $\hat{\mu}=\hat{x}$ are

$$
\begin{aligned}
\frac{\Gamma_{x y z}}{\Gamma_{\mathrm{bg}}}= & \frac{3}{4 k} \int_{0}^{k} d k_{z} \frac{k_{z}^{2}}{k^{2}}\left(1+\frac{4 \kappa^{2}}{k^{2}-k_{z}^{2}}\right) \\
& \times \frac{\left(\sqrt{k^{2}+\kappa^{2}-k_{z}^{2}}-\kappa\right)^{2}}{k^{2}+2 \kappa^{2}-k_{z}^{2}} \rho, \\
\frac{\Gamma_{x y}}{\Gamma_{\mathrm{bg}}}= & \frac{3 \pi}{2 k} \sum_{n} \frac{k_{z n}^{2}}{k^{2} R_{n}}\left(1+\frac{4 \kappa^{2}}{k^{2}-k_{z n}^{2}}\right) \\
\frac{\Gamma_{x y z}^{\prime}}{\Gamma_{\mathrm{bg}}}= & \frac{3}{4 k} \int_{0}^{k} d k_{z} \rho^{\prime}, \quad \frac{\Gamma_{x y}^{\prime}}{\Gamma_{\mathrm{bg}}}=\frac{3 \pi}{2 k} \sum_{n} \frac{1}{k_{n}^{\prime}}, \\
\frac{\Gamma_{z}}{\Gamma_{\mathrm{bg}}}= & \frac{6 \pi \kappa^{2}-k_{z n}^{2}}{\left(4 k^{2}-k^{2}\right.}, \\
\frac{\Gamma_{0}^{2}}{\Gamma_{\mathrm{bg}}}= & \frac{\left.6 d^{2}\right) k^{3}}{4 \chi^{2}-d^{2}} \sum_{n} \frac{k_{z n}^{2}}{k_{n}^{4} R_{n}} \delta\left(k-k_{n}\right) .
\end{aligned}
$$

Primed symbols refer to contributions of $p$-type modes. The $k_{z}$-dependent functions $\sigma, \rho, \rho^{\prime}$ in these equations are 


$$
\begin{gathered}
\sigma=\frac{\sin p a}{\sin k_{z} a}, \quad \rho=\frac{\sigma\left(k_{z}\right)}{1+\xi k_{z} / \tan k_{z} a}, \\
\rho^{\prime}=\frac{\sigma^{\prime}\left(k_{z}\right) S\left(p^{\prime}\right) / R\left(p^{\prime}\right)}{1+\xi k^{2} /\left(k_{z} \tan k_{z} a\right)},
\end{gathered}
$$

and $R_{n}, R(p)$, and $S\left(p^{\prime}\right)$ are given by Eqs. (34), (28), and (C4). The expression for $\sigma^{\prime}\left(k_{z}\right)$ is the same as $\sigma\left(k_{z}\right)$ but with the dependence of $p$ on $k_{z}$ given by Eqs. (26) and (B4) instead. The wave vector of a $1 \mathrm{D}$ mode is given by $k_{1}$ $=\sqrt{k^{2}+8 /(2|\chi|-d)^{2}}$ as in Eq. (21) and $\kappa$ is a function of $k_{z}$ given by Eq. (16). The spontaneous emission rate $\Gamma_{z}$ vanishes if $k_{z}$ lies inside a band gap. The total emission rates for a $\hat{z}$ and $\hat{x}$ dipole given by the sum of the expressions in Eqs. (36) and (37) are plotted as a function of $k$ in Fig. 7 for a specific example of the most interesting case $\chi<0$. For $\Gamma_{z}$ there is a concentration of the odd wave functions $h(z)$ at the bottom of the band gaps. This gives the narrow peaks in the left curves of Fig. 7. Spontaneous emission turns out to be predominantly in the same direction as the dipole moment. We attribute this unusual behavior to the evanescent nature of the field modes.

Because the bound modes of our model are exact, they give the $\delta$-function contribution in Eq. (37). In practice, there will be a finite fraction $\beta$ of light emitted into a fully bound mode at resonance. The width of the cavity resonances, which is zero in our case, is determined by dissipation of light inside the layers and possible deviations from the model in the overlap regions of the layers. The calculation of the linewidth of the bound states, the spontaneous emission factor $\beta$, and the laser threshold for a system with an active layer are discussed in [13].

\section{CONCLUSIONS}

We identified a class of dielectric structures that are threedimensional cavities for the optical field. In the absence of dissipation these cavities have exact bound states. The structures generally consist of several layers with anisotropic dielectric tensors, placed at right angles with respect to each other. Localization in the three dimensions is obtained as a combination of waveguiding and Bragg reflection. This requires periodic structures in at least one dimension. The simplest realization, shown in Fig. 2, consists of two layers that are placed at right angles and a stack of layers in the third direction, which resembles a VCSEL. The Bragg reflectors localize the waves in the vertical direction inside the crossed waveguide, but also localize the evanescent waves. This results in the bound states of our system.

Because the cavity resonances in our structures are determined only by loss and not by leakage, the linewidths may be quite narrow. We expressed the resonance frequencies of the bound modes in terms of the cavity dimensions $a, b, d$ and the dielectric constants $\varepsilon_{1}, \varepsilon_{2}$ that characterize the layers. We evaluated spontaneous emission into the other modes of the radiation field. Due to the "evanescent-wave" nature of most cavity modes, spontaneous emission occurs predominantly in the direction parallel to the dipole moment of

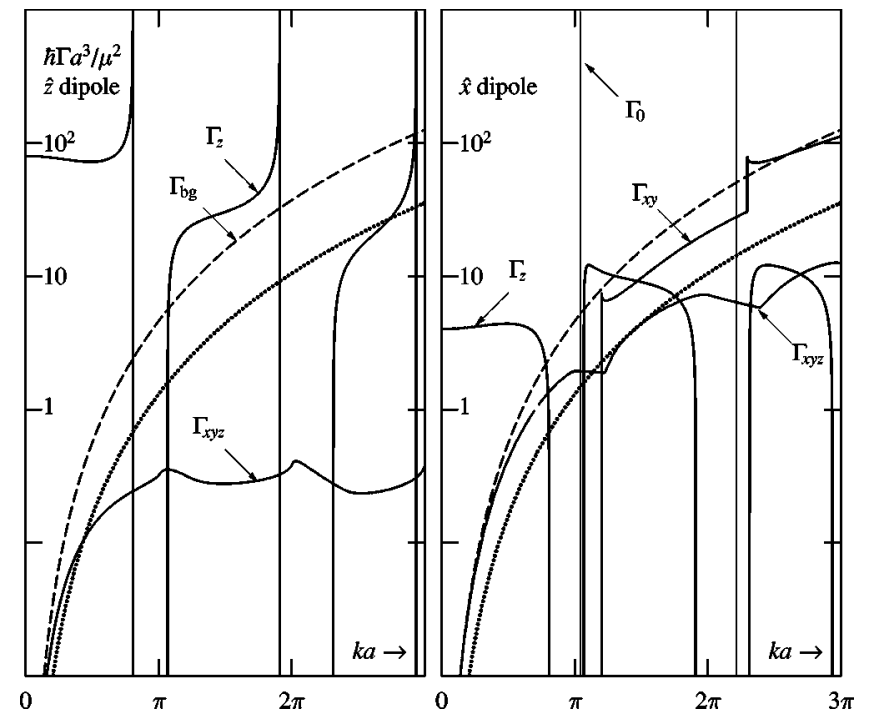

FIG. 7. Partial emission rates. On the left, the emission rates for a vertical dipole; on the right, emission rates for a horizontal dipole. The curves for $\Gamma_{z}$ have band gaps. The value of $\Gamma_{x y}$ is zero below the frequency corresponding to the first bound state. The $\delta$ peaks for bound states are indicated by the vertical lines. The dashed and dotted curves are the background and free-space emission rates. The curves are obtained with Eqs. (36) and (37) for the case $\varepsilon_{1}=4$, $\varepsilon_{2}=1, d=a=4 b$, so that $\chi=-3 a / 4, \xi=-3 a / 16$.

the emitter, instead of orthogonal to it. The bound states have a small mode volume (of the order of a few cubic wavelengths), so that the coupling to an emitter placed in the center of the mode can be strong when the cavity dimensions are chosen optimally for the specific transition frequency. Photons emitted from the central region are likely to end up in the bound state and the noise from the random emission in other modes will then be relatively small. To demonstrate the existence of bound states in a dielectric system as proposed here, one might envisage the case of microwaves with a centimeter-sized model. Microscopic realizations of the proposed structures may be promising for future cavity QED experiments $[23,24]$.

\section{ACKNOWLEDGMENTS}

This work is part of the research program of the Stichting voor Fundamenteel Onderzoek der Materie (FOM), which is financially supported by the Nederlandse Organisatie voor Wetenschappelijk Onderzoek (NWO).

\section{APPENDIX A: NORMALIZATION OF BLOCH STATES}

We here consider the general case of a one-dimensional periodic potential $W(z)$ with period $a$. The stationary solutions of the scalar equation (7) can be simultaneous eigenstates of the translation operation over the lattice period $a$. The Bloch states, which we shall denote by $h_{n}(p, z)$, are defined by the requirement that the states obtain a phase factor $e^{i p a}$ upon translation. We therefore have

$$
W(z+a)=W(z), \quad h_{n}(p, z+a)=e^{i p a} h_{n}(p, z)
$$


The Bloch momentum $p$ is a periodic variable modulo period $2 \pi / a$. Because the Bloch waves are also periodic in the spatial coordinate $z$ modulo the lattice period $a$, when multiplied by $e^{-i p z}$, there exist two different Fourier expansions. In order to get a convenient normalization, we must consider the Fourier expansions of the derivative of the Bloch states. We write

$$
\begin{aligned}
\frac{-i}{k_{z}} \frac{d}{d z} h_{n}(p, z) & =\sum_{l=-\infty}^{\infty} e^{i l p a} \widetilde{c}_{n}(z-l a) \\
& =e^{i p z} \sum_{l=-\infty}^{\infty} e^{i l q z} c_{n}(p+l q) .
\end{aligned}
$$

Note that the coefficients in these expansions in waves with period $l$ are expressed in terms of continuous functions $\tilde{c}_{n}$ and $c_{n}$ for energy band $n$. The function $\tilde{c}_{n}(z)$ is called the Wannier function and is the Fourier transform of $c_{n}\left(p_{z}\right)$ :

$$
\tilde{c}_{n}(z)=\frac{1}{q} \int_{-\infty}^{\infty} d p_{z} e^{i p_{z} z} c_{n}\left(p_{z}\right) .
$$

The Bloch and Wannier waves are normalized with

$$
\begin{aligned}
& \int_{0}^{a} d z\left|h_{n}(p, z)\right|^{2}[1+W(z)]=\int_{-\infty}^{\infty} d z \tilde{c}_{n}^{2}(z) \\
&=\frac{a}{q} \int_{-\infty}^{\infty} d p_{z} c_{n}^{2}\left(p_{z}\right)=\frac{1}{q}, \\
& \int_{-\infty}^{\infty} d z h_{n^{\prime}}^{*}\left(p^{\prime}, z\right) h_{n}(p, z)[1+W(z)]=\delta_{n^{\prime} n} \delta\left(p^{\prime}-p\right) .
\end{aligned}
$$

This normalization for the Bloch states is consistent with the $\delta$-function normalization given in Eq. (12).

For the potential $W(z)$ given in Eq. (23), the Wannier wave function can be explicitly calculated in the momentum representation. For small $b$, this wave function is given by

$$
c_{n}\left(p_{z}\right)=\frac{\xi / a}{\sqrt{2 \pi R_{n}\left(p_{z}\right)}} \frac{p_{z} k_{z n}\left(p_{z}\right)}{p_{z}^{2}-k_{z n}^{2}\left(p_{z}\right)} .
$$

The momentum $p_{z}$ is not restricted to one Brillouin zone, but ranges from $-\infty$ to $+\infty$.

\section{APPENDIX B: THE $p$-TYPE MODE FUNCTIONS}

We complete our analysis of the cavity modes by discussing the $p$-type polarization modes. These are needed for the calculation of the spontaneous emission rates in Sec. IV. The $p$-type modes are of the form

$$
\begin{gathered}
\vec{E}^{\prime}(\vec{r})=\frac{1}{\sqrt{\mathcal{R}^{\prime}}}\left(k_{y} \hat{x}-k_{x} \hat{y}\right) f^{\prime}(x) g^{\prime}(y) h^{\prime}(z), \\
\mathcal{R}^{\prime}=\left(k^{2}-k_{z}^{2}\right) \varepsilon_{1} .
\end{gathered}
$$

The primes on all these symbols only refer to the fact that we are dealing here with $p$-type modes, in contrast to the corre- sponding unprimed quantities in the main text that refer to $s$-type modes. Since the polarization lies in the $x y$ plane, the $p$-polarized modes are decoupled from the crossed vertical planes. The transverse behavior is essentially freely propagating. The normalized solutions of $f^{\prime}(x)$ and $g^{\prime}(y)$ that are even and odd in the coordinates are therefore given by

$$
\begin{gathered}
f^{\prime+}(x)=\sqrt{1 / \pi} \cos k_{x} x, \\
f^{\prime-}(x)=f^{-}(x)=\sqrt{1 / \pi} \sin k_{y} y, \\
g^{\prime+}(y)=\sqrt{1 / \pi} \cos k_{y} y, \\
g^{\prime-}(y)=g^{-}(y)=\sqrt{1 / \pi} \sin k_{y} y .
\end{gathered}
$$

By substitution of the form (B1) in Maxwell's equation (2) one obtains the following wave equation for the function $h^{\prime}(z)$ :

$$
-\frac{d^{2}}{d z^{2}} h^{\prime}(z)=k_{z}^{2} h^{\prime}(z)+k^{2} W(z) h^{\prime}(z) .
$$

The solutions $h^{\prime}(z)$, the dispersion relation, the energy bands, and the discrete solutions for $k_{z}$ are given by the same expressions as Eqs. (25), (26), (29), (30), and (32) but with the angle $\phi_{z}$ replaced by $\phi_{z}^{\prime}$. This phase shift for $p$-polarized modes is defined by

$$
\tan \phi_{z}^{\prime}=\xi k^{2} / 2 k_{z}, \quad-\pi / 2<\phi_{z}^{\prime}<\pi / 2 .
$$

In the first energy band, labeled with $n=1$, the dispersion relation (26) does not allow for a real solution of $k_{z}$ in the range $\left|p^{\prime}\right| a \leqslant \arccos \left(1-\xi k^{2} a / 2\right)$, when $\xi>0$ and $\xi k^{2} a \leqslant 4$. In this range $k_{z}$ is imaginary. If $\xi k^{2} a>4$, the entire first band has imaginary $k_{z}$. Although these Bloch states are locally constructed from evanescent waves, they have a propagating character, because $p^{\prime}$ is real and belongs to a continuous band of eigenvalues.

Although Eq. (B3) has the form of a Schrödinger equation, the normalization of Bloch waves $h_{n}^{\prime}(p, z)$ and Wannier functions $c_{n}^{\prime}(z)$ must also be given by the normalization condition Eq. (A1) of a Helmholz equation, in order to obtain the correct normalization of Eq. (9) for the field modes. The normalization constant $R_{n}(p)$ in Eq. (25) of the continuum modes (for both $\alpha=0, \alpha=\xi$ ) are generally given by

$$
\begin{gathered}
R_{n}(p)=1+\left(\frac{1}{\tan k_{z} a}-\frac{1}{k_{z} a}\right) \tan \phi_{z}+\frac{\xi}{a}, \\
R_{n}=\left(\frac{1}{\sin ^{2} k_{z n} a}+\frac{1}{k_{z n} a \tan k_{z n} a}\right) a+\frac{2 \xi}{\tan ^{2} k_{z n} a} .
\end{gathered}
$$

These expressions reduce to Eqs. (28) and (34) only when $\tan \phi_{z}=\xi k_{z} / 2$.

The different mode types are listed in Fig. 8. Generally, the degeneracy of the modes is related to the number of directions in which a mode is spatially extended, by deg $=2^{\mathrm{dim}}$. The modes with imaginary $k_{z}$ but real $p$ are an exception. These modes are extended along the $z$ axis inside the cavity but do not propagate in the exterior region where there are no more layers. In this region outside the cavity, which is 


\begin{tabular}{|c|c|ccc|c|c|cccc|}
\hline pol & idx & $k_{x}$ & $k_{y}$ & $k_{z}$ & $p$ & $\mathrm{dm}$ & $\mathrm{dg}$ & $<-d<-d / 2 \geq-d / 2>0$ \\
\hline$s$ & $x y z$ & re re re re & 3 & 8 & $\sqrt{ }$ & $\sqrt{ }$ & $\sqrt{ }$ & $\sqrt{ }$ \\
$s$ & $y z$ & im re re re & 2 & 4 & $\sqrt{ }$ & & $\sqrt{ }$ \\
$s$ & $x z$ & re im re re & 2 & 4 & $\sqrt{ }$ & & \\
$s$ & $x y$ & re re re im & 2 & 4 & $\sqrt{ }$ & $\sqrt{ }$ & $\sqrt{ }$ & $\sqrt{ }$ \\
$s$ & $y$ & im re re im & 1 & 2 & $\sqrt{ }$ & & & $\sqrt{ }$ \\
$s$ & $x$ & re im re im & 1 & 2 & $\sqrt{ }$ & & & $\sqrt{ }$ \\
$s$ & $z$ & im im re re & 1 & 2 & $\sqrt{ }$ & $\sqrt{ }$ & \\
$s$ & 0 & im im re im & 0 & 1 & $\sqrt{ }$ & $\sqrt{ }$ & \\
\hline$p$ & $x y z$ & re re re re & 3 & 8 & $\sqrt{ }$ & $\sqrt{ }$ & $\sqrt{ }$ & $\sqrt{ }$ \\
$p$ & $x y z$ & re re im re & 3 & 4 & $\sqrt{ }$ & $\sqrt{ }$ & $\sqrt{ }$ & $\sqrt{ }$ \\
$p$ & $x y$ & re re re im & 2 & 4 & $\sqrt{ }$ & $\sqrt{ }$ & $\sqrt{ }$ & $\sqrt{ }$ \\
$p$ & $x y$ & re re im im & 2 & 4 & $\sqrt{ }$ & $\sqrt{ }$ & $\sqrt{ }$ & $\sqrt{ }$ \\
\hline
\end{tabular}

FIG. 8. Different types of mode for the different intervals of the effective 2D susceptibility $\chi=\left(\varepsilon_{2}-\varepsilon_{1}\right) d / \varepsilon_{1}$. The first and second columns contain the polarization ( $s$ or $p$ type) and the index (directions of propagation). Columns four and five contain the number of dimensions in which the mode is propagating and the degeneracy. The last column indicates which types occur in the intervals $(-\infty,-d)$, which requires material with a negative dielectric constant, $[-d,-d / 2),[-d / 2,0]$, and $(0, \infty)$ for $\chi$. The $p$-type modes with imaginary $k_{z}$ exist for $\xi>0$.

not treated in this paper, solutions with imaginary $k_{z}$ must decay exponentially. This implies that there exists only one solution for $h(z)$ instead of two.

\section{APPENDIX C: CALCULATION OF EMISSION RATES}

We here give the expressions of the electric field components in $\hat{z}$ and $\hat{x}$ at the origin for all mode types. The spontaneous emission rate is given by Eq. (35). The intensities of the $s$ - and $p$-type modes in the $z$ and $x$ directions are found from Eqs. (3) and (B1) as

$$
\begin{gathered}
\left|\hat{z} \cdot \vec{E}_{\vec{k}}(\vec{r})\right|^{2}=\frac{1}{\varepsilon_{1}} \frac{\left|k^{2}-k_{z}^{2}\right|}{\left|k_{z}\right|^{2} k^{2}} f_{k_{x}}^{2}(x) g_{k_{y}}^{2}(y)\left|\frac{d}{d z} h_{k_{z}}(z)\right|^{2}, \\
\left|\hat{z} \cdot \vec{E}_{\vec{k}}^{\prime}(\vec{r})\right|^{2}=0, \\
\left|\hat{x} \cdot \vec{E}_{\vec{k}}(\vec{r})\right|^{2}=\frac{1}{\varepsilon_{1}} \frac{k_{z}^{2}}{k^{2}} \frac{1}{\left|k^{2}-k_{z}^{2}\right|}\left(\frac{d}{d x} f_{k_{x}}(x)\right)^{2} \\
\times g_{k_{y}}^{2}(y) h_{k_{z}}^{2}(z), \\
\left|\hat{x} \cdot \vec{E}_{\vec{k}}^{\prime}(\vec{r})\right|^{2}=\frac{1}{\varepsilon_{1}} \frac{k_{y}^{2}}{k^{2}-k_{z}^{2}} f_{k_{x}}^{\prime 2}(x) g_{k_{y}^{\prime}}^{\prime 2}(y) h_{k_{z}^{\prime}}^{\prime 2}(z) .
\end{gathered}
$$

The extended and localized wave functions $f(x), g(y)$, $f^{\prime}(x)$, and $g^{\prime}(y)$ are given in Eqs. (15), (17), and (B2). At the origin,

$$
\begin{gathered}
f_{k_{x}}^{+2}(0)=\frac{1}{\pi} \frac{k_{x}^{2}}{k_{x}^{2}+\kappa^{2}}, \quad\left(\frac{d}{d x} f_{k_{x}}^{+}\right)^{2}(0)=\frac{1}{\pi} \frac{k_{x}^{2} \kappa^{2}}{k_{x}^{2}+\kappa^{2}}, \\
f_{k_{x}}^{-2}(0)=0, \quad\left(\frac{d}{d x} f_{k_{x}}^{-}\right)^{2}(0)=\frac{k_{x}^{2}}{\pi}, \\
f^{2}(0)=\frac{\kappa}{|1+\chi \kappa|}, \quad\left(\frac{d}{d x} f\right)^{2}(0)=\frac{\kappa^{3}}{|1+\chi \kappa|} .
\end{gathered}
$$

The expressions for $g_{k_{y}}^{+2}(0), \quad\left(d g_{k_{y}}^{+} / d y\right)^{2}(0), g_{k_{y}}^{-2}(0)$, $\left(d g_{k_{y}}^{-} / d y\right)^{2}(0)$, and $g^{2}(0),(d g / d y)^{2}(0)$ are the same, but with $f, k_{x}$ replaced by $g, k_{y}$. According to Eqs. (25) and (33), the behavior of the wave functions $h$ of the extended and localized modes at $z=0$ are given by

$$
\begin{gathered}
h_{k_{z}}^{+2}(0)=\frac{\sigma^{2}\left(k_{z}\right)}{\pi R(p)} \frac{\tan k_{z} a}{2 \tan \phi_{z}+\tan k_{z} a}, \\
\left(\frac{d}{d z} h_{k_{z}}^{+}\right)^{2}(0)=0, \\
h_{k_{z}}^{-2}(0)=0, \quad\left|\frac{d}{d z} h_{k_{z}}^{-}\right|^{2}(0)=\frac{\sigma^{2}\left(k_{z}\right) k_{z}^{2}}{\pi R(p)}, \\
h^{2}(0)=\frac{2}{R_{n}}, \quad\left(\frac{d}{d z} h\right)^{2}(0)=0,
\end{gathered}
$$

with Eqs. (27), (28), and (34). The function $\sigma\left(k_{z}\right)$ $=\sin p a / \sin k_{z} a$ is introduced for compact notation. For $h_{k_{z}}^{\prime 2}(0)$, the same expression holds as for $h_{k_{z}}^{+2}(0)$, with $\sigma\left(k_{z}\right), R(p)$, and $\phi_{z}$ replaced by $\sigma^{\prime}\left(k_{z}\right), R\left(p^{\prime}\right)$, and $\phi_{z}^{\prime}$ given by Eqs. (B4), (B5), and (B6). It follows that a vertical dipole couples only to $s$-type modes with even or bound $f(x)$ and $g(y)$, and with odd $h(z)$. A horizontal dipole couples to both the $s$ - and $p$-type modes. At least one of the two wave functions $f(x)$ or $g(y)$ and also $h(z)$ must be either even or localized.

The calculation of the partial emission rates given by Eq. (35) for a 3D mode and analogous equations for the other modes starts by substitution of Eqs. (C1), (C2), and (C3). The integration over $k_{x}$ and $k_{y}$ can be performed explicitly using cylindrical coordinates. The third integral over the quasimomentum $p$ can be transformed into an integral over the energy bands $k_{z}$, using the relations

$$
\begin{gathered}
\frac{d k_{z}}{d p}=\frac{\sigma\left(k_{z}\right)}{R(p)}, \quad \frac{d k_{z}}{d p^{\prime}}=\frac{\sigma^{\prime}\left(k_{z}\right)}{S\left(p^{\prime}\right)}, \\
S\left(p^{\prime}\right)=1+\left(\frac{1}{\tan k_{z} a}-\frac{1}{k_{z} a}\right) \tan \phi_{z}^{\prime} .
\end{gathered}
$$


These relations can be verified with Eqs. (26) and (28). The results of the integrations are given by Eqs. (36) and (37).

If $\chi<-d$ there exist more 2D and 1D modes, as is indicated in Fig. 8. For this case one finds additional emission rates, for a dipole $\hat{\mu}=\hat{z}$,

$$
\frac{\Gamma_{x z}}{\Gamma}=\frac{\Gamma_{y z}}{\Gamma}=\frac{3}{k^{3}} \int_{k_{2}}^{\infty} d k_{z} \frac{\kappa}{|1+\chi \kappa|} \sqrt{k^{2}+\kappa^{2}-k_{z}^{2}} \sigma,
$$

and for a dipole $\hat{\mu}=\hat{x}$,

$$
\begin{aligned}
\frac{\Gamma_{x z}}{\Gamma}= & \frac{3}{k^{3}} \int_{k_{2}}^{\infty} d k_{z} \frac{\kappa}{|1+\chi \kappa|} \frac{k_{z}^{2}}{k_{z}^{2}-k^{2}} \frac{k^{2}+3 \kappa^{2}-k_{z}^{2}}{k^{2}+2 \kappa^{2}-k_{z}^{2}} \\
& \times \sqrt{k^{2}+\kappa^{2}-k_{z}^{2}} \rho, \\
\frac{\Gamma_{x}}{\Gamma}= & \frac{6 \pi}{k^{3}} \sum_{n} \frac{\kappa}{|1+\chi \kappa| R_{n}} \frac{k_{n z}^{2}}{k_{n z}^{2}-k^{2}} \frac{k^{2}+3 \kappa^{2}-k_{z n}^{2}}{k^{2}+2 \kappa^{2}-k_{z n}^{2}} \\
& \times \sqrt{k^{2}+\kappa^{2}-k_{z n}^{2}},
\end{aligned}
$$

$$
\begin{aligned}
& \frac{\Gamma_{y z}}{\Gamma}=\frac{3}{k^{3}} \int_{k_{2}}^{\infty} d k_{z} \frac{\kappa^{3}}{|1+\chi \kappa|} \frac{k_{z}^{2}}{k_{z}^{2}-k^{2}} \frac{\sqrt{k^{2}+\kappa^{2}-k_{z}^{2}}}{k^{2}+2 \kappa^{2}-k_{z}^{2}} \rho, \\
& \frac{\Gamma_{y}}{\Gamma}=\frac{6 \pi}{k^{3}} \sum_{n} \frac{\kappa^{3}}{|1+\chi \kappa| R_{n}} \frac{k_{z n}^{2}}{k_{z n}^{2}-k^{2}} \frac{\sqrt{k^{2}+\kappa^{2}-k_{z n}^{2}}}{k^{2}+2 \kappa^{2}-k_{z n}^{2}} .
\end{aligned}
$$

Here, one must substitute for $\kappa$ the expressions for the 2D modes and 1D modes given in Eqs. (18) and (20), respectively. The lower boundary value in the integration over $k_{z}$ and the summation over $k_{z n}$ follows from Eq. (22) as $k_{2}$ $=\sqrt{k^{2}+4 /(|\chi|-d)^{2}}$. Band gaps must be excluded from the integration.

These expressions are also valid for the 2D and 1D modes that occur for $\chi>0$, provided that the interval $\left[k_{2}, \infty\right]$ for the integration of $k_{z}$ and the summation of the discrete solutions $k_{z n}$ is replaced by the interval $[0, k]$.
[1] J.M. Gérard, B. Sermage, B. Gayral, B. Legrand, E. Costard, and V. Thierry-Mieg, Phys. Rev. Lett. 81, 1110 (1998).

[2] D. Deppe, L. Graham, and D. Huffaker, IEEE J. Quantum Electron. 35, 1502 (1999).

[3] A. Eschmann and C.W. Gardiner, Phys. Rev. A 49, 2907 (1994).

[4] V. Sandoghdar, F. Treussart, J. Hare, V. Lefèvre-Seguin, J.-M. Raimond, and S. Haroche, Phys. Rev. A 54, R1777 (1996).

[5] S.C. Ching, H.M. Lai, and K. Young, J. Opt. Soc. Am. B 4, 1995 (1987); 4, 2004 (1987).

[6] P.T. Leung, S.Y. Liu, and K. Young, Phys. Rev. A 49, 3057 (1994); E.S.C. Ching, P.T. Leung, and K. Young, in Optical Processes in Microcavities, edited by R.K. Chang and A.J. Campillo (World Scientific, Singapore, 1996), p. 1.

[7] E. Yablonovitch, Phys. Rev. Lett. 58, 2059 (1987).

[8] P.R. Villeneuve, S. Fan, and J.D. Joannopoulos, Phys. Rev. B 54, 7837 (1996).

[9] P.W. Anderson, Phys. Rev. 109, 1493 (1958); Philos. Mag. B 52, 505 (1985).

[10] S. John, Phys. Rev. Lett. 53, 2169 (1984).

[11] A. Lagendijk, M.P. van Albada, and M.B. van der Mark, Physica A 104, 183 (1986).
[12] E.T. Jaynes and F.W. Cummings, Proc. IEEE 51, 89 (1963).

[13] P.M. Visser, K. Allaart, and D. Lenstra (unpublished).

[14] R.J. Glauber and M. Lewenstein, Phys. Rev. A 43, 467 (1991).

[15] W. Vogel and D.-G. Welsch, Lectures on Quantum Optics (Akademie Verlag, Berlin, 1994).

[16] P.M. Visser and G. Nienhuis, Opt. Commun. 136, 470 (1997).

[17] T.J. Shepherd, P.J. Roberts, and R. Loudon, Phys. Rev. E 55, 6024 (1997).

[18] N.W. Ashcroft and N.D. Mermin, Solid State Physics (HoltSaunders, Philadelphia, 1976).

[19] E.M. Purcell, Phys. Rev. 69, 681 (1946).

[20] H. Morawitz, Phys. Rev. Lett. 18, 1792 (1969).

[21] P.W. Milonni and P.L. Knight, Opt. Commun. 9, 119 (1973).

[22] D.G. Deppe, C. Lei, C.C. Lin, and D.L. Huffaker, J. Mod. Opt. 41, 325 (1994).

[23] R. Jin, D. Boggavarapu, M. Sargent, P. Meystre, H.M. Gibbs, and G. Khitrova, Phys. Rev. A 49, 4038 (1994).

[24] Y. Yamamoto, S. Machida, and G. Björk, Phys. Rev. A 44, 657 (1991); Y. Yamamoto, S. Machida, Y. Horikoshi, K. Igeta, and G. Björk, Opt. Commun. 80, 337 (1991); G. Björk, H. Heitmann, and Y. Yamamoto, Phys. Rev. A 47, 4451 (1993). 